\title{
Spontaneous Symmetry Breaking in Finite Systems
}

\author{
James D. Fraser*†
}

The orthodox characterization of spontaneous symmetry breaking (SSB) in statistical mechanics appeals to novel properties of systems with infinite degrees of freedom, namely, the existence of multiple equilibrium states. This raises the same puzzles about the status of the thermodynamic limit fueling recent debates about phase transitions. I argue that there are prospects of explaining the success of the standard approach to SSB in terms of the properties of large finite systems. Consequently, despite initial appearances, the need to account for SSB phenomena does not offer decisive support to claims about the explanatory and representational indispensability of the thermodynamic limit.

1. Introduction. In both classical and quantum theory, systems with infinite degrees of freedom can have properties that are not found in any finite system. There has recently been much debate among philosophers of physics about the role these novel properties of infinite systems play in accounting for important physical phenomena.

In statistical mechanics, phase transitions, such as the boiling of water, are associated with nonanalyticities in the free energy that only occur in the thermodynamic (or macroscopic) limit. Roughly speaking, this means taking the volume and particle number to infinity, while systems that actually display phase behavior, such as kettles of water, are evidently finite in extent. Nevertheless, Batterman (2005) claims that the nonanalyticities that occur in the thermodynamic limit are needed to successfully represent and

Received August 2015; revised October 2015.

*To contact the author please write to: School of Philosophy, Religion and History of Science, University of Leeds, Woodhouse Lane, Leeds, LS2 9JT, UK; e-mail: phjdf @1eeds.ac.uk.

$\dagger$ I would like to thank Aernout Van Enter, Klaas Landsman, and Hal Tasaki for useful correspondence and three anonymous referees for comments on earlier versions of the article. Thanks also to Steven French and Laura Ruetsche for crucial encouragement.

Philosophy of Science, 83 (October 2016) pp. 585-605. 0031-8248/2016/8304-0007\$10.00

Copyright 2016 by the Philosophy of Science Association. All rights reserved. 
explain phase transitions. Similarly, Ruetsche (2003) argues that unitarily inequivalent representations found in the thermodynamic limit of quantum statistical mechanics play an essential role in accounting for phase structure in quantum systems and need to be taken physically seriously.

This view of the thermodynamic limit has been contested by Butterfield (2011), Norton (2012), and Callender and Menon (2013), however. According to these authors, the thermodynamic limit is predictively and explanatorily successful in phase transition theory because of features infinite systems share with large finite systems - specifically, the nonanalytic functions found in the limit are said to provide a good approximation to the values of macroscopic quantities in realistic, finite volume models. On this view, phase transitions in concrete systems are sharp changes in their macroscopic properties, rather than physical discontinuities.

This article examines a phenomenon that raises very similar interpretive issues, namely, spontaneous symmetry breaking (SSB). As with phase transitions, the definition of SSB in statistical mechanics refers to properties afforded by the thermodynamic limit: in this case, the nonuniqueness of the system's equilibrium state. At first glance, this characterization of SSB lends new support to claims about the indispensability of the thermodynamic limit. There are seemingly inescapable arguments to the effect that the characteristic features of SSB phenomena cannot occur in finite systems, and consequently the kind of deidealization strategy pursued by Butterfield and others in the phase transition debate is foiled. I argue here that this is not in fact the case: there are prospects of making sense of the success of the standard approach to SSB in terms of the behavior of large finite systems. The implications of this program for our understanding of the notion of SSB, and philosophical issues with which it has become embroiled, are discussed.

2. Spontaneous Symmetry Breaking in Statistical Mechanics. The notion of SSB is employed in a number of theoretical contexts, not always without controversy. Understanding the nature of SSB in quantum field theory (QFT), and the appeal to spontaneously broken gauge symmetry in standard presentations of the Higgs mechanism in particular, is an ongoing project in the foundations of physics. ${ }^{1}$ My focus here, however, is on the more mundane SSB phenomena described by statistical mechanics, the archetype being ferromagnetism. At high temperatures the electron spins inside a ferromagnetic crystal are uncorrelated, and the magnetization is effectively zero, but if the temperature is lowered to some critical value, spins suddenly align,

1. See Friederich (2013) and references therein for recent critiques of the notion of spontaneously broken gauge symmetry, especially in the standard model. I bracket concerns about gauge symmetry breaking here, restricting my attention to the breaking of nongauge symmetries in statistical mechanics. 
giving rise to a net magnetization in a particular direction. Although the electromagnetic interactions that produce these aligned states are isotropic, below the critical temperature the ferromagnet displays stable states that are not rotationally symmetric.

It will be useful for what follows to distinguish two assumptions underlying of the standard definition of SSB in statistical mechanics. First, there is the symmetry breaking part. The emergence of asymmetric behavior at low temperatures is taken to mean that the system's equilibrium macrostate below the critical temperature is noninvariant under the relevant symmetry transformation (a global rotation in the case of the ferromagnet). Second, there is the spontaneous part. The sense in which this symmetry is broken spontaneously is typically spelled out via a contrast with so-called explicit symmetry breaking. ${ }^{2}$ This describes situations in which asymmetric behavior is produced by asymmetries in the underlying dynamics. Applying an external magnetic field to a ferromagnet, for instance, gives rise to aligned states, but in this case the rotational symmetry of interactions between spins is explicitly broken by the external field, which takes the form of a term in the system's Hamiltonian that is noninvariant under rotations. SSB is said to occur in the absence of any such noninvariant terms: in other words, the symmetry that is broken at low temperatures is taken to be an exact symmetry of the dynamics.

Taking these two claims together leads, inexorably, to the identification of SSB with the degeneracy of a system's equilibrium states and, just as inexorably, to the need to take the thermodynamic limit. In general, any transformation that leaves a system's Hamiltonian invariant maps its equilibrium states into other equilibrium states. Consequently, an equilibrium state can only be noninvariant under an exact symmetry of the dynamics if it is one of a set of equilibrium states related by the action of the relevant transformation. It turns out, however, that the equilibrium state of a system with a finite number of degrees of freedom is always unique; degenerate equilibrium states are only found in the thermodynamic limit. Indeed, while it is SSB in quantum theory that is often said to require infinite degrees of freedom, the thermodynamic limit plays an analogous, and equally crucial, role in modeling SSB in quantum and classical statistical mechanics. I explain the basic structure of this account with reference to the classical Ising model here, returning to additional issues raised by quantum theory in section 4.2.

In this famous model one takes a cubic (or, more generally, hypercubic) lattice, with $N$ sites and volume $V$, and associates with each site $i$ a variable $s_{i}= \pm 1$. The values of $s_{i}$ can be interpreted as a naive representation of the orientation of spins inside a ferromagnetic crystal: $s_{i}=1$ corresponding to a

2. See Castellani (2003) for a discussion of the distinction between spontaneous and explicit symmetry breaking. 
spin aligning 'up'; $s_{i}=-1$ corresponding to a spin aligning 'down'. In keeping with this picture, we can identify the quantity, $m=\sum_{i} s_{i} / V$, with the (volume averaged) magnetization of the system. ${ }^{3}$ The basic Hamiltonian consists of a single term representing short-ranged interactions that make it energetically favorable for spins to align parallel with one another:

$$
H=-J \sum_{i, j} s_{i} s_{j},
$$

where $J$ is a positive constant parameterizing the interaction strength and the sum is taken over 'nearest neighbor' spin pairs. The key property of this Hamiltonian for our purposes is that it is invariant under a 'spin flip' transformation $s_{i} \rightarrow-s_{i}$ : it is this reflection symmetry that is spontaneously broken at low temperature.

The system's equilibrium state is represented by a probability distribution over its microstates, $S_{V}=\left(s_{1}, \ldots, s_{N}\right)$, known as the canonical ensemble:

$$
P\left(S_{V}\right)=\frac{\mathrm{e}^{-\beta H\left(S_{V}\right)}}{Z},
$$

where $\beta=1 / k_{B} T$ is the inverse temperature and $Z$ is the partition function. This distribution is clearly unique, and, in fact, the canonical ensemble assigns a unique equilibrium state to any system with finite degrees of freedom. Things become more interesting in the thermodynamic limit however. Taking $N, V \rightarrow \infty$, with $N / V$ held constant, generates an infinite lattice with a divergent total energy, so (2) is no longer a well-defined probability distribution. In the classical context the canonical ensemble is typically generalized to such infinite systems by means of the Gibbs measure formalism. ${ }^{4}$ Roughly speaking, a Gibbs measure is a probability measure over a system's microstates that behaves locally like the canonical ensemble - in the case of the Ising model, any finite subregion of the lattice is stipulated to have a probability distribution defined over its associated microstates that takes the form of (2). Stated precisely, this definition agrees with the canonical ensemble for systems with finite degrees of freedom, but infinite systems also admit Gibbs measures in this sense.

3. A note on terminology is perhaps needed here. In statistical mechanics the magnetization is often identified with the expectation value of the quantity I have called $m$. Part of the reason for this is that $\langle m\rangle$ and $m$ effectively coincide in the thermodynamic limit, as fluctuations vanish. It will be crucial to my discussion in sec. 4 to keep these quantities distinct, however, and I refer to the latter quantity as the magnetization here.

4. Presentations of the Gibbs measure formalism, and its application to SSB and phase transitions, can be found in Georgii (1988) and Lebowitz (1999). 
In fact, there can be a set of distinct Gibbs measures in the thermodynamic limit. An elegant argument (originally due to Peierls) illustrates how this can occur in the case of the Ising model on a two-dimensional lattice. ${ }^{5}$ Strictly speaking, the Hamiltonian of a finite Ising model should include an additional term representing the effects of external spins at the boundary of the lattice. If we take all of the spins at this boundary to be 'up', it can be shown that, below some critical temperature, the expectation value of $s_{i}$ at the center of the lattice is positive no matter how large $V$ is. This means that when we take $V \rightarrow \infty$ the boundary goes to spacial infinity while the expected magnetization of the system converges to a positive value. Running the same argument with 'down' spins at the boundary, we find that there are two distinct Gibbs measures, $P_{+}$and $P_{-}$, on the infinite lattice that assign positive and negative values to the magnetization, respectively. Normalized linear combinations of these measures are also Gibbs measures of the system. If we take the thermodynamic limit of a finite lattice with boundary conditions that are invariant under spin flips, ${ }^{6}$ for instance, the Gibbs measure converges to $1 / 2 P_{+}+$ $1 / 2 P_{-}$. Only $P_{+}$and $P_{-}$satisfy the properties required of genuine equilibrium states, however: in general, systems with infinite degrees of freedom can have a convex set of Gibbs measures whose extremal elements correspond to macroscopically distinct equilibrium states of the system. ${ }^{7}$

The following picture of SSB phenomena emerges. At high temperatures the Ising model has a single Gibbs measure in the thermodynamic limit corresponding to a macrostate with zero magnetization that respects the reflection symmetry of the Hamiltonian. Below the critical temperature, however, the system will enter one of the equilibrium states associated with the $P_{+}$or $P_{-}$measure in which spins align in a particular direction, breaking this symmetry. More generally, a symmetry is said to be spontaneously broken at low temperatures if there is a set of distinct equilibrium macrostates in the thermodynamic limit that are mapped into each other under the relevant transformation. A spontaneously broken rotational symmetry, for instance, as in the case of a more realistic ferromagnetic system, is associated with an infinite set of equilibrium states related to one another by rotations. Although there are differences in its formal implementation (described in sec. 4.2), this account of SSB survives essentially unchanged in quantum statistical mechanics.

5. See Kindermann and Snell (1980), and references therein, for the details. Note that this argument does not go through for a one-dimensional classical Ising model, and rigorous results are, for the most part, not available in three or more dimensions.

6. In practice, this typically means free or periodic boundary conditions; see Kindermann and Snell (1980, 34-35).

7. For a discussion of the properties of extremal Gibbs measures that justify equating them with equilibrium states, see Georgii $(1988,119)$. 
This approach to modeling SSB raises interpretive problems, as we will soon see, but as a piece of first-order science its success is undeniable. ${ }^{8}$ The asymmetric equilibrium states that appear in the thermodynamic limit give an accurate quantitative description of the macroscopic properties of concrete systems at low temperatures. Furthermore, this account clearly gets something right about the qualitative character of SSB phenomena. The availability of multiple equilibrium states at low temperatures, each on an equal footing with respect to the dynamics, captures the most intuitive sense in which ferromagnetic symmetry breaking, and analogous behavior, is 'spontaneous', namely, that the system has an equal chance of 'choosing' one of a number of possible asymmetric configurations at the critical temperature. The philosophically interesting question is not whether the infinite systems obtained in the thermodynamic limit are genuinely predictive and explanatory, but why.

Before addressing this issue in earnest, it is worth touching on the close connections with the way that phase transitions are represented in statistical mechanics. As has already been mentioned, phase transitions are associated with nonanalyticities in macroscopic observables, and ultimately the free energy, which only occur in the thermodynamic limit. The expected magnetization of the infinite Ising model, for instance, is a discontinuous function of the external magnetic field, $h$, below the critical temperature, corresponding to the first-order phase transition observed in real ferromagnets when the direction of an external field is reversed (fig. 1). The appearance of these nonanalyticities is intimately related to the nonuniqueness of the system's equilibrium state at the phase boundary. In the case of the Ising model, it is the existence of the $P_{+}$and $P_{-}$equilibrium states that allows the expected magnetization to change discontinuously at $h=0$. Indeed, the nonuniqueness of a system's equilibrium states is sometimes used as an alternative definition of a phase transition (Lebowitz 1999). It is not surprising, therefore, that SSB and phase transitions pose similar conceptual problems. Nevertheless, the standard account of SSB does raises new issues in the debate about the interpretive status of the thermodynamic limit, as I argue in the next section.

3. Interpreting the Thermodynamic Limit. There is something puzzling about the approach to SSB just described. The infinite volume models ob-

8. But, it is worth bearing in mind that the standard account of SSB has limitations that may be important for assessing its interpretive significance. An infinite system cannot always be constructed by taking the volume to infinity in the manner sketched heremany systems, especially those with long-ranged interactions, do not have a well-defined thermodynamic limit. If such systems display SSB phenomena, this will clearly be problematic for the thesis (discussed below) that the thermodynamic limit plays an indispensable role in accounting for this behavior. 


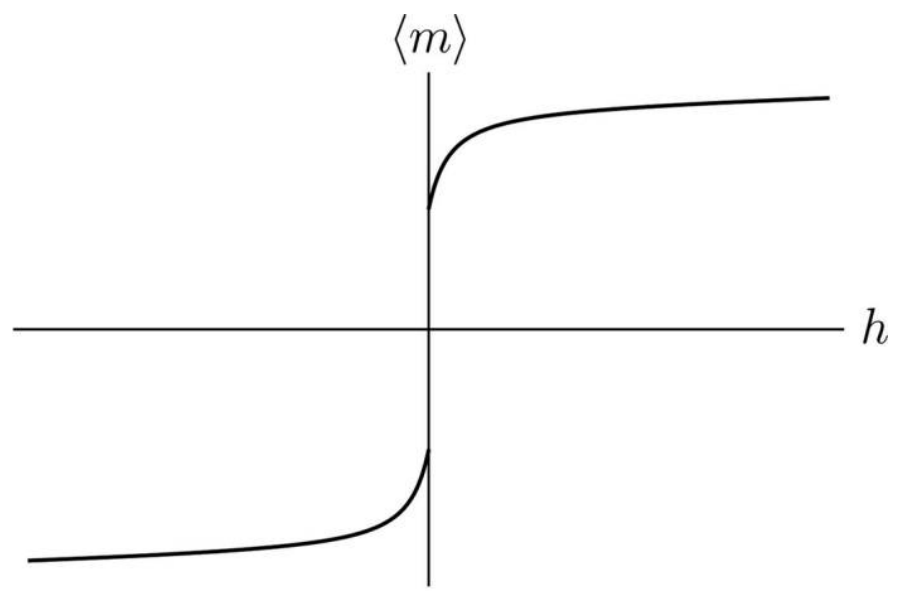

Figure 1. Expected magnetization as a function of the external magnetic field, $h$, for an Ising model on an infinite two-dimensional lattice.

tained in the thermodynamic limit seem to be highly idealized representations of the concrete systems that exhibit SSB phenomena-real ferromagnets clearly have a finite volume and number of constituents. But the explicit appeal to novel properties of infinite systems in the standard definition of SSB seems to problematize an intuitive picture of how idealized models relate to their targets. In calculating the electric field produced by a long charged wire, one often treats it as being infinitely long. Insofar as this model is predictive and explanatory, it is presumably because it captures relevant features of the target system that are preserved when this infinite length idealization is corrected - we can easily verify in this case that the electric field associated with an infinite charged wire provides a good approximation to the field close to a long finite length wire. The idea that idealized models are successful because the behavior they aim to describe is robust under 'deidealization' is most famously associated with McMullin's notion of Galilean idealization (McMullin 1985). The role of the thermodynamic limit in the standard approach to SSB does not seem to fit this framework, however. The degenerate equilibrium states that signal the onset of SSB completely disappear if we remove the thermodynamic limit and return $N$ and $V$ to realistic finite values.

As I have stressed, this is essentially the same problem engendered by the appeal to nonanalyticities in the free energy in phase transition theory. As the debate about this case shows, there are a number of ways that one might respond.

Some philosophers have concluded that the thermodynamic limit plays an indispensable role in representing and explaining physical phenomena. 
There are a number of ways that this claim might be spelled out. Batterman and others take the thermodynamic limit to be an instance of a so-called ineliminable or essential idealization that is not amenable to the kind of deidealization analysis sketched above for the infinite wire model. ${ }^{9}$ In particular, Batterman (2005) claims that, despite its status as an idealization, the nonanalyticities afforded by the thermodynamic limit correspond to genuine physical discontinuities that manifest during a change of phase. The analogous claim in the case of SSB would presumably be that the degenerate equilibrium states found in the thermodynamic limit are real features of macroscopic systems that can only be captured by idealized infinite volume models. Earman (2004) suggests a very different way of understanding how the thermodynamic limit could be representationally indispensable. As he points out, the new mathematical structures found in the limit of infinite volume also occur in a field theoretic context. Assuming that the world is, at base, a quantum field, we could take the novel properties that appear in the thermodynamic limit to correspond to features of this more fundamental reality that are missed by models with finite degrees of freedom. On this view the thermodynamic limit turns out not to be an idealization after all. ${ }^{10}$ While these two interpretations clearly differ in important respects, they both take novel properties afforded by the thermodynamic limit to have direct physical significance. Call this the indispensablist reading of the thermodynamic limit.

In the recent debate about phase transition theory, however, a number of philosophers have argued that a more conservative response to the puzzle is available. As I understand them, Butterfield (2011) and Callender and Menon (2013) want to say that, despite appearances, the thermodynamic limit is an ordinary idealization, not fundamentally unlike the limit of infinite length in the charged wire case. ${ }^{11}$ Note that the fact that novel properties obtain in an idealized limit does not, in itself, establish that they are indispensable in any interesting sense. The electric field produced by an infinitely long charged

9. It is this reading of the indispensability of the thermodynamic limit that is sometimes coupled with claims about emergence and the failure of reductive explanation. By contrast, Earman's appeal to field theory is thoroughly reductive in character, which suggests that the connection between the interpretive status of the thermodynamic limit and broader debates about emergence and reduction is more subtle than is sometimes recognized.

10. Although I do not press this point here, one can question whether either way of understanding the indispensability of the thermodynamic limit really accounts for its success. Appealing to the still inchoate notion of essential idealization arguably raises a new set of puzzles (see Shech 2013; Fraser 2015) and objections to invoking field theory in the phase transition debate, which apply just as forcefully in the case of SSB and can be found in Mainwood (2006, 228-31) and Butterfield (2011, 1078).

11. Norton (2012) gives a slightly different but closely allied diagnosis of the role of the thermodynamic limit in phase transition theory. 
wire has a translation symmetry that is not respected by any finite length wire, but nobody claims that this property is explanatorily essential, much less physically real. That is because the success of the infinite wire model is very plausibly accounted for by other properties that are robust when the infinite length idealization is removed. Similarly, according to these authors, it is not the novel properties of infinite systems but those they share with large finite systems that are doing the real explanatory and representational work in applications of the thermodynamic limit. Call this the deflationary reading of the thermodynamic limit.

The basic observation motivating this position in the phase transitions debate has been that, while the nonanalyticities in the free energy disappear when the thermodynamic limit is removed, this function can still approximate the changes in macroscopic properties of a finite system. Recall that, below the critical temperature, the expected magnetization of the Ising model becomes a discontinuous function of the external magnetic field in the thermodynamic limit, signaling a first-order phase transition (fig. 1). General theoretical considerations, and evidence from numerical simulations, suggest that the expected magnetization of a finite Ising model has an abrupt change in sign at $h=0$, which becomes increasingly steep as $N$ increases, so that, if the lattice is sufficiently large, it will be very well approximated by the discontinuous magnetization function of an infinite Ising lattice. We seem to have a simple schema for explaining the success of the orthodox approach to phase transitions in terms of the behavior of large finite systems: a real phase transition is a sharp but smooth change in a large system's macroscopic properties that is approximated by the nonanalytic free energy found in the infinite volume limit to accuracies within acceptable error.

There is a great deal more to be said about this dispute. Perennial philosophical questions about reduction, idealization, and scientific explanation loom large in the debate, and the need to account for the universal behavior observed in disparate systems during continuous phase transitions raises further puzzles about the explanatory role of the thermodynamic limit. ${ }^{12}$ The focus of this article, however, is on what SSB brings to the table.

The account of SSB detailed in the previous section presents a new challenge for the deflationary reading of the thermodynamic limit and seems to provide stronger grounds for claims about its indispensability. Pointing to the fact that nonanalytic functions can approximate analytic ones does not help in this case. The issue now is not why we can legitimately model a ferromagnet's macroscopic properties as changing discontinuously under var-

12. Batterman has long argued that the role of the thermodynamic limit in renormalization group explanations of the universality of critical exponents is not susceptible to deidealization; see, e.g., Batterman (2010). See also Callender and Menon (2013) for an attempt to develop a deflationary reading of the thermodynamic limit in this context. 
iations in the external magnetic field but why, in the absence of an external magnetic field, the system can be successfully represented as having multiple available equilibrium states at low temperatures, despite the fact that the equilibrium state of any finite system is unique. Not only has a putative deidealization story for the thermodynamic limit not been provided in the case of SSB, there seem to be powerful reasons why no such account can be given even in principle. As we have seen, if a system's equilibrium state is unique it must be invariant under the same transformations as its Hamiltonian. This means that the expected values of macroscopic observables must be consistent with these symmetries; the expected magnetization of a finite Ising model, for instance, is always zero no matter how large the lattice is, which appears to flatly contradict what we see in real ferromagnets. Prima facie then, the description of SSB afforded by the thermodynamic limit cannot approximate behavior that is already seen in large finite systems, as they simply lack the resources needed to accommodate stable symmetry breaking macrostates.

As a result, it appears that the indispensablist reading of the thermodynamic limit is vindicated: if the sort of deidealization account supplied in the phase transition debate is ruled out, it seems that the novel properties of infinite systems appealed to in the standard approach to SSB must be taken physically seriously, in one way or another, in order to account for its success.

4. Spontaneous Symmetry Breaking in Finite Systems. This kind of nogo argument against the possibility of providing a deidealization story for the thermodynamic limit in the standard account of SSB is misleading, however, as I now argue. SSB behavior only appears to be impossible in finite systems if the modeling assumptions implicit in the standard definition of SSB are treated as sacrosanct. Relaxing the requirement that the system's equilibrium state at low temperatures is asymmetric (the symmetry breaking part), or the demand that the broken symmetry is exact (the spontaneous part), allows us to see how systems with finite degrees of freedom can exhibit the kind of behavior observed in real ferromagnets. Section 4.1 explores the former route. I point out that a system with a unique, symmetric, equilibrium state can still be expected to exhibit asymmetric states over long time periods. This suggests one way of treating the thermodynamic limit as an ordinary idealization that can be made fairly precise in the case of some simple classical systems. Section 4.2 introduces the challenges quantum theory raises for understanding how SSB can occur in finite systems - I argue that they are not as insurmountable as is sometimes thought. Section 4.3 explores the latter route. Landsman (2013) has developed an approach to SSB in finite quantum systems based on the instability of their equilibrium states under asymmetric perturbations to the dynamics. The upshot of the discussion is that, despite ini- 
tial pessimism, there is a viable program for explaining the success of the standard approach to SSB in terms of the properties of finite systems.

4.1. Approach 1: Long-Lived Asymmetric States. The fact that a finite system's equilibrium state is invariant under the symmetries of its Hamiltonian does not, in fact, rule out SSB type behavior: an equilibrated finite system can still be expected to exhibit asymmetric states over very long time periods. Once again, the classical Ising model provides a useful reference point here. The canonical ensemble of the Ising model assigns a probability distribution, $P(m)$, to the possible magnetizations of the system. Provided that the boundary conditions imposed on a finite lattice do not break the interaction's reflection symmetry, $P(m)$ will also respect this symmetry, which, as has already been noted, implies that the expected magnetization is zero. This does not mean, however, that $P(m)$ must take the form of a normal distribution centered at $m=0$; it can have maxima at positive and negative $m$. The system would then be expected to spend most of its time in magnetized microstates.

In the case of the Ising model on a two-dimensional lattice, it can be shown that this is exactly what happens below the critical temperature. As was mentioned in section 2 , if the boundary conditions are suitably symmetric the Gibbs measure of the system is an equal linear combination of the $P_{+}$and $P_{-}$measures. These extremal measures assign probability one to $m= \pm a$ (for some positive $a$ ) in the thermodynamic limit and can be shown to satisfy central limit theorems as $N \rightarrow \infty . .^{13}$ This means that, below the critical temperature, $P(m)$ has two 'humps' at $m \pm a$, which become increasingly sharply peaked as $N$ increases, finally converging to Dirac measures at $N=\infty$ (fig. 2). Although rigorous results are less forthcoming, for more realistic models there are general theoretical grounds for believing that analogous behavior will hold whenever the Gibbs measure is nonunique in the thermodynamic limit. The generic result that statistical fluctuations scale like $1 / \sqrt{N}$ (where $N$ is now the number of constituents) implies that the canonical ensemble of a large finite system will have most of its mass in microstates that assign values to macrostate variables close to those associated with the degenerate equilibrium states that appear in the limit.

Consequently, we can expect such systems to spend long time periods in symmetry breaking states. A large finite Ising model below the critical temperature will enter one of the two regions of its state space in which $m \approx \pm a$, with equal probability, and remain there for a very long time. In fact, we can give heuristic arguments - ultimately based on artificial implementations of the system's dynamics, like the so-called Glauber dynamics — that a finite

13. See Kindermann and Snell (1980, 34-62) and references therein. 


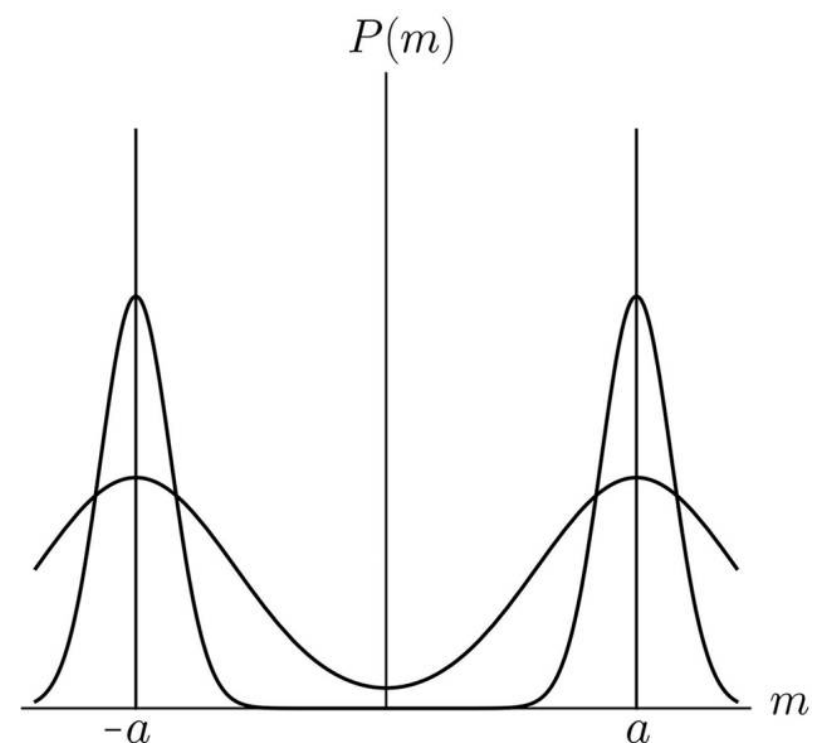

Figure 2. $P(m)$ below the critical temperature for an Ising model with small, large, and infinite $N$.

Ising model will transition between states of positive and negative magnetization with a time period proportional to $\exp \sqrt{N}$ (see Kindermann and Snell 1980, 55-61; Lebowitz 1999, 353). In the thermodynamic limit, the system will remain in an 'up' or 'down' aligned state forever - they become distinct equilibrium states. But if $N$ is of the order of Avogadro's number, the system can still be expected to exhibit a magnetization in a particular direction for a very long time indeed: much longer than we can feasibly observe real ferromagnets and perhaps longer than the age of the universe.

This points to an explanation of the successful appeal to infinite systems in the standard account of SSB in terms of properties they share with large finite systems. On this view, what we actually observe in real ferromagnets at low temperatures are long-lived asymmetric states whose macroscopic properties are well approximated by the degenerate equilibrium states found in the thermodynamic limit. The picture of SSB afforded by the thermodynamic limit, in which the system must choose between a set of symmetry breaking equilibrium states, thus provides an appropriate description of the behavior of large systems over long, but finite, time periods. ${ }^{14}$

14. In fact, this way of understanding how the standard account of SSB relates to the behavior of finite systems is sometimes found in the statistical physics literature. Commenting on the nonuniqueness of the Gibbs measure of the Ising model in the thermo- 
4.2. Finite Quantum Systems. As has already been alluded to, SSB in quantum theory has often been thought to provide additional reasons for attaching special interpretive significance to systems with infinite degrees of freedom. The question whether the approach to SSB in finite systems just sketched in the context of classical systems can be extended to quantum statistical mechanics is, therefore, an urgent one.

In many respects, the way that SSB is modeled in quantum statistical mechanics is analogous to the classical case. The canonical ensemble of a finite quantum system is traditionally represented by a density operator,

$$
\hat{\rho}=\frac{\mathrm{e}^{-\beta \hat{H}}}{Z},
$$

which, again, is not well defined in the thermodynamic limit. In the quantum context, the algebraic formulation of quantum statistical mechanics provides a rigorous framework for generalizing the canonical ensemble to infinite systems (see Bratteli and Robinson 2003; Ruetsche 2011). The analogue of Gibbs measures are so-called KMS (Kubo-Martin-Schwinger) states, which agree with (3) for finite systems but can also exist in the thermodynamic limit. As in the classical case, the canonical ensemble of a finite system is unique, but a system with infinite degrees of freedom can have a convex set of KMS states whose extremal elements correspond to distinct symmetry breaking equilibrium states. ${ }^{15}$

There are peculiarities of quantum theory, however, that do seem to have important consequences for the possibility of SSB behavior in finite systems. While classical systems with finite degrees of freedom admit multiple ground states, the corresponding quantum systems typically do not. The classical Ising model has two ground states: one with all spins pointing 'up', the other with all spins pointing 'down'. But the quantum Ising model (with a transverse magnetic field) has a single ground state, which is a superposition of the states $\psi_{+}$and $\psi_{-}$corresponding to spins aligned 'up' and 'down', respectively. ${ }^{16}$ More generally, a unique quantum ground state can typically be con-

dynamic limit, Lebowitz writes: "This means physically that when $V$ is very large the system with 'symmetric' [boundary conditions] will, with equal probability, be found in either the '+ state' or the '- state'. Of course as long as the system is finite it will 'fluctuate' between these two pure phases, but the 'relaxation times' for such fluctuations grows exponentially in $|V|$, so the either/or description correctly captures the behavior of macroscopic systems" $(1999,353)$. A similar discussion is found in Binney et al. $(1992,48-51)$.

15. There has been some debate about how best to characterize SSB in quantum statistical mechanics; see Emch and Lui (2005) and Ruetsche (2011). My discussion here is neutral with respect to this dispute.

16. In order to obtain a nontrivial quantum version of the Ising model, a transverse magnetic field term has to be added, so the basic Hamiltonian is $\hat{H}=-J \Sigma_{i, j} \hat{\sigma}_{i}^{z} \hat{\sigma}_{j}^{z}-h_{x} \Sigma_{i} \hat{\sigma}_{i}^{x}$, 
structed from a superposition of quantum states associated with degenerate minima in the potential if the system has finite degrees of freedom. In the limit of infinite degrees of freedom, however, unitarily inequivalent Hilbert space representations of the system's algebra of observables occur, which can support distinct ground and equilibrium states. In the thermodynamic, $\psi_{+}$and $\psi_{\text {- become genuine ground states belonging to unitarily inequivalent repre- }}$ sentations of the infinite quantum Ising model. This has led Ruetsche (2011) to argue that the need to accommodate SSB phenomena in quantum theory compels us to imbue multiple unitarily inequivalent representations with physical content.

The foundational issues raised by the quantum ground state and unitarily inequivalent representations are legion, particularly in the context of QFT. Recall that my ambitions here are limited to understanding the success of the standard account of SSB in statistical mechanics. The key question then is whether the uniqueness of the quantum ground state blocks the extension of the approach to SSB in finite systems (described in the previous section) to quantum systems.

Intuitively, it might seem to: surely at low temperatures the system will remain close to its ground state, and no symmetry breaking behavior will be observed. This is not as obvious as it seems, however. Finite quantum systems can support asymmetric states that are stable over very long time periods, despite the uniqueness of their ground states, as can be seen in the case of the quantum Ising model. If the potential of a finite system has multiple minima, the associated asymmetric quantum states can typically be expressed as superpositions of the ground and first excited states: in the quantum Ising model, $\psi_{ \pm}=(1 / \sqrt{2})\left(\psi_{0} \pm \psi_{1}\right)$. What happens as $N \rightarrow \infty$ is that the energy gap between these states vanishes. In the case of the quantum Ising model, it can be shown that the energy gap between the ground and first excited states, $\Delta E$, decays like $\exp (-N)$, and Koma and Tasaki (1994) prove analogous results for a broader class of lattice models that display degenerate ground states in the thermodynamic limit. This means that, if the system is large, these asymmetric states will have a very slow time evolution. A quantum Ising model that is prepared in the $\psi_{+}$state will remain in a magnetized state for an arbitrarily long time, for arbitrarily large $N$. In fact, the existence of these slowly precessing asymmetric states has been put forward in the physics literature as a way of understanding how quantum systems can exhibit SSB, most notably in Anderson's famous discussion of the electric dipole moment of ammonia and sugar (1972). ${ }^{17}$

$\left.\overline{\text { where }\left\{\hat{\sigma}^{x}\right.}, \hat{\sigma}^{y}, \hat{\sigma}^{z}\right\}$ are Pauli matrices. Henceforth, I refer to this model simply as the 'quantum Ising model'.

17. See also Anderson $(1997,175-82)$ for a similar discussion of finite quantum ferromagnetic systems. 
This suggests that the macroscopic properties of a large quantum system can be well approximated by the symmetry breaking equilibrium states found in the thermodynamic limit over long time periods, despite the uniqueness of the system's ground state. How this claim can be developed in detail is not entirely clear, however - there remains the question of how the system enters an asymmetric state in the first place and why we do not see the true quantum ground state. ${ }^{18}$ It may be that the canonical ensemble of a finite quantum system that displays degenerate equilibrium states in the thermodynamic limit has most of its mass in symmetry breaking pure states at low temperatures, and the deidealization story sketched above for the classical Ising model can be straightforwardly extended to quantum systems. ${ }^{19}$ But it has been suggested by some authors that one must appeal to additional resources, specifically the system's coupling to the environment, to account for the occurrence of SSB behavior in finite quantum systems. The next section examines an alternative approach to SSB in finite systems in this vein that has been explicitly developed in the context of quantum statistical mechanics.

4.3. Approach 2: Instability under Asymmetric Perturbations. Landsman (2013) has put forward a different approach to SSB in finite quantum systems. It turns out that the equilibrium states of a large quantum system can be very unstable under small asymmetric perturbations to the dynamics. If we relax the assumption that the symmetry that is broken at low temperatures is exact, this provides another way of understanding how SSB phenomena can manifest in finite systems.

It can be shown that adding a small perturbation to the Hamiltonian of a large quantum Ising model that favors 'up' aligning states causes the system's ground state to abruptly shift toward $\psi_{+}$, and converge to it as $N \rightarrow$ $\infty$, with analogous statements holding for the system's equilibrium state below the critical temperature. One way to see why this occurs is to consider the effective Hamiltonian governing the ground and first excited states. In the $\psi_{ \pm}$basis,

$$
H=\left(\begin{array}{cc}
0 & -\Delta E \\
-\Delta E & 0
\end{array}\right),
$$

18. Some authors claim that there is a close connection here with the measurement problem; see Emch and Lui (2005) and Landsman (2013). This is something of a red herring in my view, however, and I keep the question of how SSB in quantum systems should be understood separate from broader interpretive issues about quantum theory.

19. To my knowledge it remains a conjecture that the kind of bifurcation seen in the canonical ensemble of the classical Ising model at low temperatures also occurs in finite quantum systems. 
where $\Delta E$ is, again, the energy difference between the ground and first excited state, which, as we have seen, decays exponentially in $N$. Consequently, any term added to the upper diagonal element, no matter how small, will dominate the effective Hamiltonian for sufficiently large $N$, shifting the ground state close to $\psi_{+}$. Of course, the same argument applies to perturbations favoring 'down' aligning spins. More generally, this type of instability under asymmetric perturbations can be expected to hold if the energy gap between ground and first excited states vanishes sufficiently quickly as $N \rightarrow \infty$. ${ }^{20}$

This provides another way of explaining the success of the standard account of SSB by way of the behavior of large finite systems. In many cases, modeling the symmetry that is broken at low temperatures as an exact invariance of the target system's dynamics is an idealization. In concrete systems, small, otherwise empirically negligible, asymmetric perturbations are very likely to be physically instantiated. In the case of ferromagnetic crystals, such perturbations might originate in the system's coupling to its environment, background magnetic fields, or defects in the lattice structure. At low temperatures these tiny asymmetries have a dramatic effect on the behavior of the system, shifting it into an equilibrium state whose macroscopic properties are very well approximated by one of the degenerate equilibrium states found in the thermodynamic limit. Given our ignorance of the details of the perturbations affecting a real ferromagnet, there is an obvious epistemic sense in which a ferromagnet has an equal chance of aligning in any particular direction, and it is appropriate to represent the system as having a number of available equilibrium states below the critical temperature.

While Landsman's discussion is grounded in quantum theory, it is plausible that this kind of approach to SSB in finite systems can also be applied to classical systems. If the expected magnetization of a large classical Ising model below the critical temperature is well approximated by the discontinuous function found in the thermodynamic limit, as Butterfield and others argue, it will be very sensitive to changes in the external magnetic field. As a result, a tiny background magnetic field will shift the system's equilibrium state into a magnetized state that is well approximated by one of the symmetry breaking Gibbs measures found in the thermodynamic limit. ${ }^{21}$ This is, of course, a much less general kind of perturbation to the dynamics than those considered by Landsman, but it does suggest that the equilibrium states of finite classical systems can display the same kind of instability prop-

20. Koma and Tasaki (1994) and Landsman (2013) show that this is the case for a number of other models, but the claim that this behavior holds whenever there are degenerate equilibrium states in the thermodynamic limit is conjectural. This issue is discussed further in sec. 5 .

21. Binney et al. (1992, 48-51) give a characterization of SSB in finite classical systems along these lines. 
erties as their quantum counterparts. SSB behavior in finite classical systems may also be produced by small asymmetries arising from environmental effects, then.

A puzzle might seem to arise here. We have now seen two, apparently quite different, ways of producing the phenomenological features of SSB phenomena in finite systems: the first based on long-lived asymmetric states and the second on asymmetric perturbations to the dynamics. If both accounts apply to the same model, how are we to understand what actually happens in a concrete system that displays SSB? There is no real difficulty here, however. Following Lui (2003) we can distinguish between the features of a model that make SSB possible and the physical mechanism that brings about asymmetric behavior in a particular system that model is used to represent. A finite volume model can display both long-lived asymmetric states and the kind of instability properties cited by Landsman, yet the way that SSB arises in different physical instantiations of the model may differ from case to case. Furthermore, there is no reason to treat these two approaches to SSB in finite systems as in competition. Each can be viewed as getting around the apparent impossibility of SSB in finite systems by rejecting the symmetry breaking and spontaneous part of the standard definition of SSB, respectively, but it is, of course, also possible to reject both. It is plausible that in many concrete system's environmental perturbations and the stability properties of asymmetric states both play a role in producing SSB type phenomena. ${ }^{22}$

5. Philosophical Implications. The picture of how SSB phenomena manifest in concrete systems that emerges from the foregoing discussion is a complex one, and many of the details remain open to further investigation. Nevertheless, the findings of the previous section have important implications for the interpretive issues raised by SSB. In particular, the suggestion that the success of the standard account of SSB compels us to reify novel properties of infinite systems has been undermined.

Recall that SSB seemed to provide a stronger case for claims about the representational indispensability of the thermodynamic limit than phase transitions. Not only does the orthodox characterization of SSB appeal to novel properties of infinite systems, there seemed to be a no-go argument against the possibility of SSB behavior in models with finite degrees of freedom that ruled out the kind of deidealization strategy pursued by Butterfield and others in the phase transition debate. I take the foregoing discussion to show that this is not the case. While SSB defined as the existence of degenerate equilibrium

22. Environmental perturbations may be responsible for shifting the system into an asymmetric state, while the stability properties of this state explain why we continue to see a symmetry breaking macrostate over a long time period. 
states cannot occur in finite systems, this does not show that the kind of behavior observed in real ferromagnets is impossible. The modeling assumptions underlying this definition, which I dubbed the symmetry breaking and spontaneous part, ought not to be treated dogmatically. If a broader view is taken, and these assumptions are weakened in various ways, we can see how SSB behavior can occur in finite systems.

More than this, though, we have seen that there are prospects of accounting for the predictive and explanatory success of infinite volume systems in the standard approach to SSB in terms of features they share with large finite systems. There is clearly work still to be done here. The results discussed in this article have, for the most part, been limited to the Ising model. It is ultimately a conjecture that the key statements about large finite models can be generalized to the full range of systems to which the standard account of SSB is applied. Furthermore, for quantum systems in particular, there remain open questions about what form the resulting account can be expected to take. Much the same can be said of the account supplied in the phase transition debate, however - the claim that the free energy function found in the thermodynamic limit approximates that of a large finite system is based on heuristic arguments and has not been shown to hold in general. In both cases, once a framework for deidealizing the thermodynamic limit is on the table the claim that novel properties of infinite systems must be treated as physically real in order to make sense of the relevant physics loses its bite. If the standard account of SSB is to be used to support claims about the physical significance of novel properties of infinite models, reasons need to be given for thinking that the program put forward in section 4 cannot account for the predictive and explanatory success of the thermodynamic limit in this context. ${ }^{23}$

This is not, of course, to say that the indispensablist reading of the thermodynamic limit has been refuted here. While the approach discussed in

23. There are two main ways that indispensablists might proceed here. First, they might argue that the approach developed in sec. 4 does not recover the right explanandum - the fact remains that finite systems cannot accommodate behavior that is both 'symmetry breaking' and 'spontaneous' in the strict sense introduced in sec. 2. If this kind of objection is to go through, however, reasons need to be given for thinking that the concrete phenomena that stand in need of explanation, like ferromagnetism, actually satisfy these requirements, beyond the fact that the standard approach defines SSB in this way, which simply begs the question. Second, they might try to find successful applications of the standard account of SSB in which the strategies put forward in sec. 4 fail. The spontaneous breaking of gauge symmetries might seem to present a possible avenue of attack along these lines. It is not clear that we can make sense of gauge symmetries being broken by environmental perturbations, as in Landsman's approach, for instance. As noted in $\mathrm{n}$. 1, however, the notion of spontaneously broken gauge symmetry is itself controversial. Whether pressure can be put on the deflationary reading by delving into this issue remains open to further investigation. 
this article offers defenders of the deflationary reading a way of responding to the challenge posed by SSB, and thereby weakens one prima facie motivation for the indispensablist position, this is not, in itself, an argument against it. Furthermore, as I have emphasized, the dispute over the interpretive status of the novel properties of infinite systems is a multifaceted issue that cannot be settled by examining a single application of the thermodynamic limit.

With this caveat in mind, I close by considering the consequences of embracing a deflationary reading of the thermodynamic limit for our understanding of SSB and related issues. I have argued that there are two, mutually compatible, ways to accommodate symmetry breaking behavior in finite systems, corresponding to the rejection of the symmetry breaking and spontaneous part of the standard definition of SSB. Both routes seem to have revisionary implications. On the first approach the relevant symmetry is not really broken in the system's full-time evolution. While a finite Ising system can display magnetized states over long time periods, as $t \rightarrow \infty$ it will spend an equal amount of time in 'up' and 'down' aligned states, consistent with the fact that the expected magnetization is zero. The second approach, however, muddies the conventional distinction between spontaneous and explicit symmetry breaking. Explicit symmetry breaking, you will recall, refers to asymmetric behavior produced by asymmetries in the dynamics, which is exactly what the perturbations appealed to in Landsman's approach are. ${ }^{24}$ Furthermore, in both cases the question of what counts as SSB behavior does not seem to have a precise answer. The notion of a long-lived asymmetric state is obviously a vague one, and while there is clearly a qualitative difference between the production of magnetized states by tiny environmental effects and via the application of an external magnetic field, the distinction is not sharp. Admitting that both approaches can play a role in producing symmetry breaking behavior further compounds this situation.

This result is not as radical as it might seem, however. While it may have implications for the ontic status of SSB phenomena, the deflationary reading of the thermodynamic limit, as I understand it, need not have revisionary consequences for scientific practice and certainly does not amount to an injunction that physics abandon the standard definition of SSB or cease appeals to infinite systems ${ }^{25}$ There are instructive parallels here with Callender

24. Although, as I suggested in sec. 4.3, we can plausibly still recover one sense in which the behavior of a ferromagnet is 'spontaneous' on Landsman's approach, namely, that spins have an equal chance of aligning in any particular direction.

25. It is important to distinguish the kind of deidealization program discussed in this article from attempts to offer a new definition of a phase transition in terms of the resources of finite systems surveyed in Callender and Menon (2013). I take the deflationarv reading of the thermodvnamic limit to be a claim about why infinite svstems. and 
and Menon's (2013) discussion of extensive quantities in statistical mechanics. Roughly speaking, a quantity is said to be extensive if it behaves additively when the system is divided into subsystems. Strictly speaking, no quantity satisfies this definition when the system is finite; boundary effects spoil the additivity of quantities like the entropy, and the distinction between extensive and intensive quantities only really applies in the thermodynamic limit. But, although the notion of extensivity does not carve nature at its joints, there is clearly a sense in which it is appropriate and useful to treat entropy and other observables as extensive in situations in which boundary effects are negligible. Similarly, although SSB phenomena might not form a genuine natural kind by the deflationist's lights, section 4 provides a framework for explaining why the standard definition of SSB can be fruitfully applied to a range of physical phenomena. ${ }^{26}$ Recall that the interpretive puzzle, as I originally posed it, was why the standard approach to SSB is successful. Accordingly, if successful, the deidealization program discussed in this article will justify the current practice of statistical mechanics, not undermine it.

But, revising our understanding of the nature of SSB phenomena in the manner countenanced by the deflationist may have an important knock-on effect on broader philosophical issues. I briefly touch on one here, namely, the interpretive problems posed by unitarily inequivalent representations in quantum theory. Ruetsche (2011) uses the way SSB is modeled in statistical mechanics to motivate what she calls a coalescence approach to the interpretation of quantum theory in the limit of infinite degrees of freedom. She argues that in order to explain SSB phenomena in quantum statistical mechanics we must move beyond a single Hilbert space and attribute physical content to multiple unitarily inequivalent Hilbert space representations found in the thermodynamic limit. The deflationist, of course, will deny this. If the program sketched in section 4 is taken seriously, then all of the relevant physical information about what goes on in a real ferromagnet at low temperatures can be captured by a finite quantum model and, therefore, a single Hilbert space. What this means for the larger debate about the philosophical significance of unitarily inequivalent representations is a matter for further investigation. Unitarily inequivalent representations, and the notion of $\mathrm{SSB}$, recur in the context of QFT and raise a gamut of issues that have not been touched on here. But the discussion of this article does suggest that there

definitions that appeal to them, are successful in statistical mechanics. Whether an alternative definition of SSB in finite systems can be provided, and what methodological and metaphysical implications this might have, is, I think, a further question.

26. There is clearly a great deal more to be said here. One might wonder, for instance, whether it is possible to understand the role that the notion of SSB has played in fruitful analogies between condensed matter physics and QFT within the deidealization program sketched in sec. 4. What impact, if any, the discussion of this article has on broader methodological issues of this kind is a topic for further investigation. 
is scope to push back against the claim, championed by Ruetsche, that multiple unitarily inequivalent representations must be taken ontologically seriously because of the indispensable role they play in scientific explanations.

\section{REFERENCES}

Anderson, Philip W. 1972. "More Is Different.” Science 177 (4047): 393-96.

1997. Concepts in Solids: Lectures on the Theory of Solids. Singapore: World Scientific. Batterman, Robert. 2005. "Critical Phenomena and Breaking Drops: Infinite Idealizations in Physics." Studies in Historv and Philosophy of Modern Physics 36:225-44.

- 2010. "On the Explanatory Role of Mathematics in Empirical Science." British Journal for the Philosophy of Science 61 (1): 1-25.

Binney, J. J., N. J. Dowrick, A. J. Fisher, and M. E. J. Newman. 1992. The Theory of Critical Phenomena: An Introduction to the Renormalization Group. Oxford: Oxford University Press.

Bratteli, Ola, and Derek Robinson. 2003. Operator Algebras and Quantum Statistical Mechanics. Vol. 2. New York: Springer.

Butterfield, Jeremy. 2011. "Less Is Different: Emergence and Reduction Reconciled.” Foundations of Phvsics 41 (6): 1065-1135.

Callender, Craig, and Tarun Menon. 2013. "Turn and Face the Strange . . . Ch-ch-changes: Philosophical Questions Raised by Phase Transitions." In The Oxford Handbook of Philosophy of Physics, ed. Robert Batterman. Oxford: Oxford University Press.

Castellani, Elena. 2003. "On the Meaning of Symmetry Breaking." In Symmetries in Physics: Philosophical Reflections, ed. Katherine Brading and Elena Castellani. Oxford: Oxford University Press.

Earman, John. 2004. "Curie's Principle and Spontaneous Symmetry Breaking." International Studies in the Philosophy of Science 18 (2-3): 173-98.

Emch, Gerard G., and Chuang Lui. 2005. "Explaining Quantum Spontaneous Symmetry Breaking." Studies in the Historv and Philosophv of Modern Phvsics 36:137-63.

Fraser, James D. 2015. "Essential Idealisations and the Nature of Scientific Explanation." Unpublished manuscript, University of Leeds.

Friederich, Simon. 2013. "Gauge Symmetry Breaking in Gauge Theories: In Search of Clarification.” European Journal for Philosophy of Science 3 (2): 157-82.

Georgii, Hans-Otto. 1988. Gibbs Measures and Phase Transitions. Berlin: De Gruyter.

Kindermann, Ross, and Laurie Snell. 1980. Markov Random Fields and Their Application. Providence, RI: American Mathematics Society.

Koma, Tohru, and Hal Tasaki. 1994. "Symmetry Breaking and Finite-Size Effects in Quantum Many-Body Systems.” Journal of Statistical Physics 76 (3-4): 745-803.

Landsman, N. P. 2013. "Spontaneous Symmetry Breaking in Quantum Systems: Emergence or Reduction?" Studies in Historv and Philosophy of Science B 44 (4): 379-94.

Lebowitz, Joel L. 1999. "Statistical Mechanics: A Selective Review of Two Central Issues." $\underline{\text { Re- }}$ views of Modern Physics 71:346-57.

Lui, Chuang. 2003. "Spontaneous Symmetry Breaking and Chance in a Classical World." Philosophy of Science 70 (3): 590-608.

Mainwood, Paul. 2006. "More Is Different? Emergent Properties in Physics.” PhilSci, University of Pittsburgh. http://philsci-archive.pitt.edu/8339/.

McMullin, Ernan. 1985. "Galilean Idealization." Studies in Historv and Philosophy of Science 16 (3): 247-73.

Norton, John D. 2012. “Approximation and Idealization: Why the Difference Matters." Philosophy of Science 79 (2): 207-32.

Ruetsche, Laura. 2003. "A Matter of Degree: Putting Unitary Inequivalence to Work." Philosophv of Science 70 (5): 1329-42.

2011. Interpreting Quantum Theories. Oxford: Oxford University Press.

Shech, Elay. 2013. "What Is the Paradox of Phase Transitions?" Philosophy of Science 80 (5): $1170-81$. 